\title{
How Important are the Primary Teeth?
}

\author{
Mohammad Karimi* \\ Department of Pediatric dentistry, Sepideh Dental Clinic, Iran
}

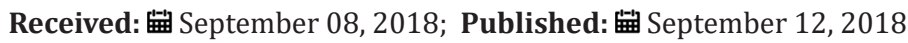

*Corresponding author: Mohammad Karimi, Department of Pediatric dentistry, Sepideh Dental Clinic, Iran

\section{Editorial}

White and beautiful pearls that appear for the first time in the sixth month on the lower gingiva are the primary teeth that erupt after one another until the age of two. These teeth should remain in the mouth without any problem until the eruption of the permanent teeth so that have the proper function. Unfortunately, many children lose their teeth much sooner than the time they should naturally fall. The most important cause of early tooth loss is caries, which is due to the lack of attention of parents to children's oral hygiene, the negligence of referral for regular examinations by the dentist to detect early caries and postponing the essential and appropriate dental treatments. Therefore, caries becomes more aggressive and causes pain and discomfort for the child. Many parents are convinced that because the primary teeth are temporary, they do not consider any kind of care for them; and even with the presence of caries, they wait so long the child has a toothache and cannot tolerate it. Therefore, they come to the dentist to extract that tooth. However, if the parents are familiar with the importance of these teeth, they will work harder to maintain them. Carious lesions in the primary teeth, in addition to the pain and discomfort that it causes, it might cause the damages to the underneath follicles of permanent teeth and would interfere with their developmental process as a result of tooth infection. In addition, chewing and nourishing of the child, which is vital in the developmental age, will be disrupted; and the child may become malnourished.
Contrary to this notion, these teeth are important for many different reasons. Primary teeth play an important role in chewing food, gaining the skills of speaking, and having a beautiful face and a child's confidence. On the other hand, having no anterior teeth can disturb his speech because, with the contact of the tongue with the anterior teeth, the pronunciation of many letters will be created. Hence, the absence of these teeth will cause problems in the child's pronunciation, which is sometimes habitually left in him. Additionally, the absence of these teeth can also eliminate the beauty and confidence of the child and make him become mocked by his peers. Most importantly, the buds of permanent teeth are located below the primary teeth, and when the time of eruption comes to pass, the root of the teeth is absorbed; the permanent teeth will replace them. If a tooth is removed from the mouth before the eruption time, space is occupied by the other teeth. As a result, there is not enough room for the permanent tooth to erupt, which causes unwanted tooth crowding. Also, these teeth act as a natural space maintainer for permanent teeth, which means that their presence will help the permanent teeth to erupt at the right time and in the right place. Extracting or early teeth loss can shorten the length of the jaw and thus causes teeth crowding in a few years later. That's where we should talk about the need for a costly orthodontic treatment in the future. Therefore, we strongly recommend by promoting oral hygiene in children, parents are able to prevent dental caries in order to preserve primary teeth.

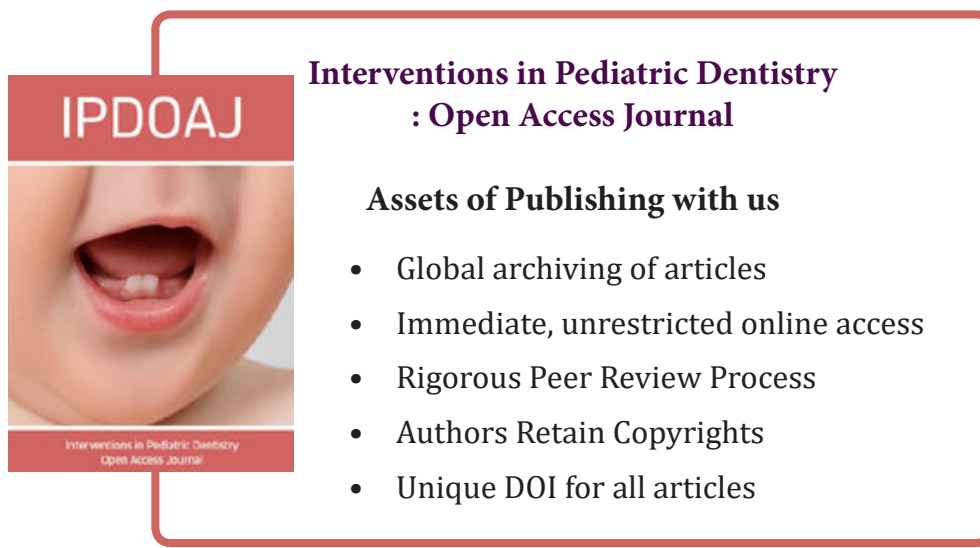

